\title{
Denosumab-related osteonecrosis of the jaw in a patient with bone metastases of prostate cancer: A case report and literature review
}

\author{
HITOSHI YOSHIMURA ${ }^{1}$, SEIGO OHBA ${ }^{2}$, HISATO YOSHIDA ${ }^{1}$, KYOKO SAITO ${ }^{1}$, \\ KAZUYOSHI INUI $^{1}$, RIE YASUI ${ }^{1}$, DAI ICHIKAWA ${ }^{1}$, MINAKO AIKI ${ }^{1}$, JUNICHI KOBAYASHI ${ }^{1}$, \\ SHINPEI MATSUDA ${ }^{1}$, YOSHIAKI IMAMURA ${ }^{3}$ and KAZUO SANO ${ }^{1}$ \\ ${ }^{1}$ Department of Dentistry and Oral Surgery, Unit of Sensory and Locomotor Medicine, \\ Division of Medicine, Faculty of Medical Sciences, University of Fukui, Fukui 910-1193; ${ }^{2}$ Department of \\ Regenerative Oral Surgery, Graduate School of Biomedical Sciences, Nagasaki University, Nagasaki 852-8588; \\ ${ }^{3}$ Division of Surgical Pathology, University of Fukui Hospital, Fukui 910-1193, Japan
}

Received June 9, 2016; Accepted March 9, 2017

DOI: $10.3892 / \mathrm{ol} .2017 .6121$

\begin{abstract}
Denosumab, a human monoclonal antibody directed against the receptor activator of nuclear factor- $\kappa \beta$ ligand (RANKL), is used for the treatment of patients with metastatic cancer of the bone or osteoporosis. Recent reports have demonstrated that denosumab can induce osteonecrosis of the jaw (ONJ), but reported cases of this are uncommon. The present study reports the case of an 86-year-old male with prostate cancer patient exhibiting bone metastases who developed ONJ whilst receiving denosumab. To elucidate the influence of denosumab on the development of ONJ, the present study also reviewed the literature, including clinical trials and case reports. In the clinical trials, the prevalence of denosumab-related ONJ was higher in patients with cancer compared with those with osteoporosis. The high risk of ONJ in patients with cancer was thought to be associated with the differing dose and frequency of denosumab administration. The prevalence of ONJ was not significantly different between patients receiving denosumab and bisphoshonate (BP). In the reported cases, denosumab-related ONJ had a similar clinical presentation to BP-related ONJ. There was also a tendency for denosumab-related ONJ to develop in the mandible of elderly patients. Previous invasive dental treatment was a commonly shared characteristic of patients with denosumab-related ONJ. A complex medical history was also suspected to affect the prevalence. No clear association between the dose or duration of denosumab treatment and the development of ONJ was
\end{abstract}

Correspondence to: Dr Hitoshi Yoshimura, Department of Dentistry and Oral Surgery, Unit of Sensory and Locomotor Medicine, Division of Medicine, Faculty of Medical Sciences, University of Fukui, 23-3 Matsuokashimoaizuki, Eiheiji, Yoshida, Fukui 910-1193, Japan

E-mail: omfs@u-fukui.ac.jp

Key words: denosumab, osteonecrosis, cancer, bone metastasis, jaw observed. Although conservative treatments are given for denosumab-related ONJ, non-improving cases were managed surgically with primarily positive results. Because denosumab may offer superior results compared with BP for the treatment of metastatic cancer of the bone or osteoporosis, the use of denosumab is expected to increase in the near future. Clinicians should also be aware of the risk factors for denosumab-related ONJ, in order to aid in its diagnosis. In addition, patients treated with denosumab should receive prophylactic treatment to maintain their oral health prior to, during and after denosumab treatment.

\section{Introduction}

Currently, the clinical management of metastatic cancer of the bone or osteoporosis targets signaling pathways associated with bone remodeling (1). For example, bisphosphonate (BP) inhibits osteoclast function and bone turnover to manage these diseases (1). Patients receiving BP treatment, including zoledronate, can develop osteonecrosis of the jaw (ONJ), specifically BP-related ONJ (BRONJ), although the relative prevalence of this is low (1). Denosumab, a human monoclonal antibody directed against the receptor activator of nuclear factor- $\kappa \beta$ ligand (RANKL), has been approved as an antiresorptive agent (1). Denosumab inhibits the binding of RANKL to RANK, thereby reducing osteoclast formation, function and survival, which results in decreased bone resorption and increased bone density (1). However, studies have demonstrated that denosumab can induce ONJ, similarly to BP $(1,2)$. There is currently insufficient evidence regarding the diagnosis and treatment of denosumab-associated ONJ.

The present study reports a case of ONJ of the maxilla in an 86-year-old male who had been treated with denosumab to manage bone metastases associated with prostate cancer. To elucidate the influence of denosumab on the development of ONJ, the present study also reviewed the literature, including clinical trials (3-10) and case reports (11-21), in regards to the prevalence, clinical characteristics and management of ONJ associated with denosumab. 


\section{Case report}

An 86-year-old male was referred to the Department of Dentistry and Oral Surgery at the University of Fukui Hospital (Fukui, Japan) in December 2013 with bone exposure in the left upper premolar region. The bone exposure had developed over a period of 1 month. The patient had a prior history of endodontic treatment of the left upper premolar teeth during 2008 (month unknown). The patient was a non-smoker and did not drink alcohol. In addition, the patient had no history of receiving radiation therapy to the head and neck region, and his medical history included tuberculosis, myocardial infarction, hypothyroidism and prostate cancer. The patient's prostate cancer had been treated with high-intensity focused ultrasound therapy in November 2002 and a radical prostatectomy in January 2004. Pathological diagnosis of the surgical specimen revealed moderately differentiated adenocarcinoma of the prostate (pT3aNOM0, stage III), and hormone therapy (combined androgen blockade therapy using an anti-androgen drug and LH-RH agonist; doses unknown) was initiated in January 2004 (duration, 4 years). Due to increasing prostate-specific antigen values, the patient was treated with chemotherapy in February 2008 (including docetaxel $40 \mathrm{mg}$ per patient triweekly and tegafur/uracil $200 \mathrm{mg} / \mathrm{day}$; duration, 4 years and 10 months); skeletal metastases were identified in January 2009. The patient had been treated once with zoledronate (4 mg per patient) in January 2009, but it was discontinued due to hypocalcemia and renal dysfunction. The patient underwent 6 subcutaneous injections of $120 \mathrm{mg}$ denosumab between September 2012 and March 2013 and 1 subcutaneous injection of $120 \mathrm{mg}$ denosumab in December 2013.

Physical examination of the patient revealed no facial swelling or neck lymphadenopathy. No paresthesia of the left infra-orbital region was evident. An intra-oral examination revealed a $5 \times 10 \mathrm{~mm}$ dehiscence of the oral mucosa in the upper left premolar region. Exposure of the underlying bone, redness and slight swelling of the surrounding gingiva were also observed; however, there was no obvious pus discharge (Fig. 1). The patient complained of tenderness in the left infraorbital area. A panoramic radiograph revealed radiolucencies with diffuse irregularity in the left maxilla and around the apices of the left upper premolar teeth (Fig. 2A). An axial computed tomography image (bone window setting) identified an area of low density in the left posterior maxilla and the destruction of the buccal cortical bone, indicating osteomyelitis (Fig. 2B). A coronal image (bone window setting) demonstrated bone sequestrum in the left maxilla, perforation in the inferior wall of the maxillary sinus and sinusitis (Fig. 2C). Bone scintigraphy revealed increased uptake of ${ }^{99 \mathrm{~m}} \mathrm{Tc}$-methylene diphosphonate (MDP) from the left maxilla to part of the sinus and provided evidence of active bone turnover (Fig. 2D). The uptake of ${ }^{99 \mathrm{~m}} \mathrm{Tc}-\mathrm{MDP}$ at the sites of the upper right humerus, left scapula, sternum, chest and lumbar spine, ribs and pelvic bone indicated multiple metastatic lesions of the bone (Fig. 2E). Over 8 weeks (from December 2013 to January 2014), the patient presented no change in the area of the exposed bone. The patient's history combined with the clinical and radiographic findings was consistent with $\mathrm{ONJ}$ and the administration of denosumab was discontinued in January 2014.

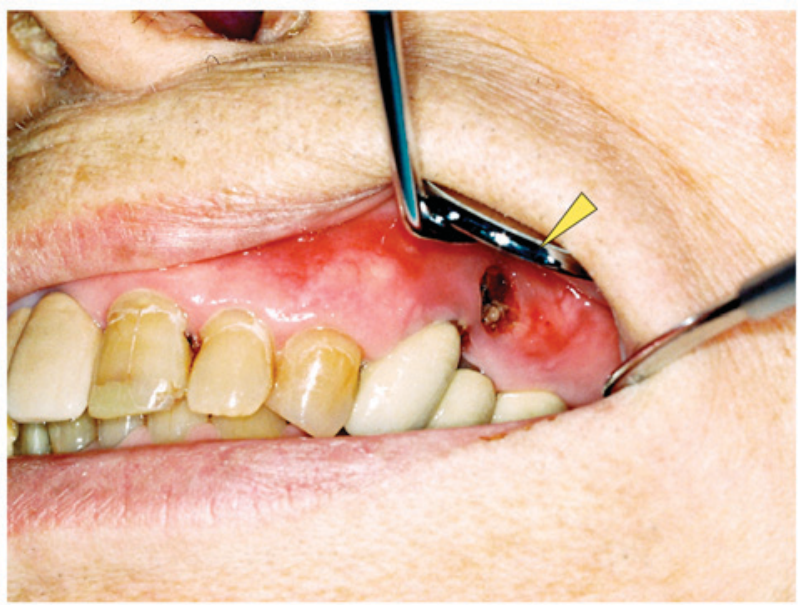

Figure 1. Clinical appearance of the denosumab-related osteonecrosis of the jaw. An intraoral view revealed exposed necrotic bone in the left upper maxilla with redness of the mucosa (indicated by the arrowhead).

The patient refused radical surgical treatment under general anesthesia, so the exposed bone was partially removed under local anesthesia. Histological examination of 5- $\mu \mathrm{m}$ sections of the surgical sample, using light microscopy and hematoxylin and eosin staining, revealed complete osteonecrosis with empty osteocytic lacunae throughout the extent of the bone fragment (Fig. 3A and B). Actinomyces colonies were observed, but no osteocytes, osteoblasts or osteoclasts were present in the histologically examined sample; there was also no sign of prostate cancer metastasis. Microbiological analysis revealed a mixed normal oral flora, including Streptococcus, Neisseria and Corynebacterium species, but Actinomyces was not identified in the microbiologically examined sample. A final diagnosis of ONJ of the maxilla was confirmed. For $>30$ months (December 2013 to May 2016, the date of writing), the patient was maintained on daily chlorhexidine mouth rinses, and an antibiotic (amoxicillin $750 \mathrm{mg} / \mathrm{day}$ for 7 days) was used during periods of exacerbation (increased pain, redness and pus-containing discharge). The intraoral dehiscence remained, but the patient did not complain of pain. The patient provided written informed consent for the use of their data in the present study.

\section{Discussion}

A relatively recent development for the treatment of metastatic cancer of the bone or osteoporosis has been the inhibition of RANKL, a member of the tumor necrosis factor superfamily of ligands (1). Denosumab is a human monoclonal antibody directed against RANKL, which inhibits the binding of RANKL to RANK, thereby inhibiting osteoclast differentiation and function (1).

ONJ is defined as exposed bone in the maxillofacial region that does not heal for $\geq 8$ weeks in patients with no history of craniofacial radiation $(2,22,23)$. ONJ was first described in patients receiving BP therapy (24), and an increasing number of patients with BRONJ have been reported. There has been a previous report that the use of denosumab can also lead to ONJ (11). The American Association of Oral and Maxillofacial Surgeons has proposed that ONJ caused by BP or denosumab 
A

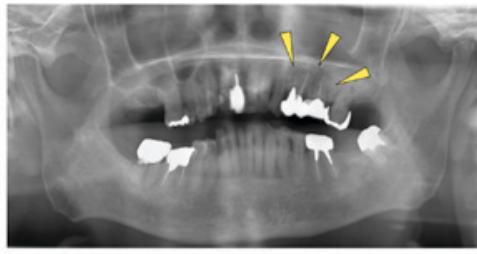

$\mathrm{D}$
$\mathrm{B}$

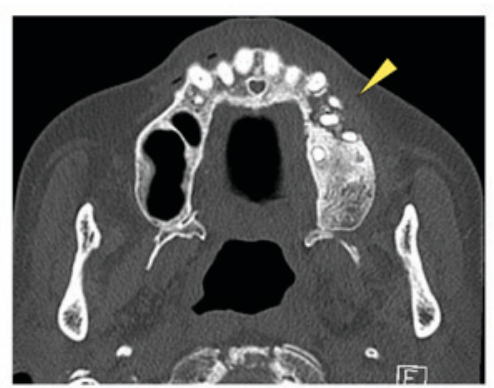

$\mathrm{E}$
$\mathrm{C}$

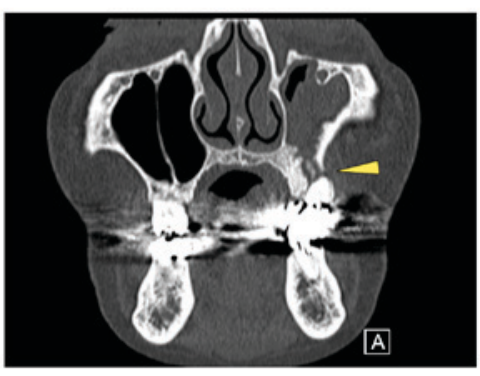

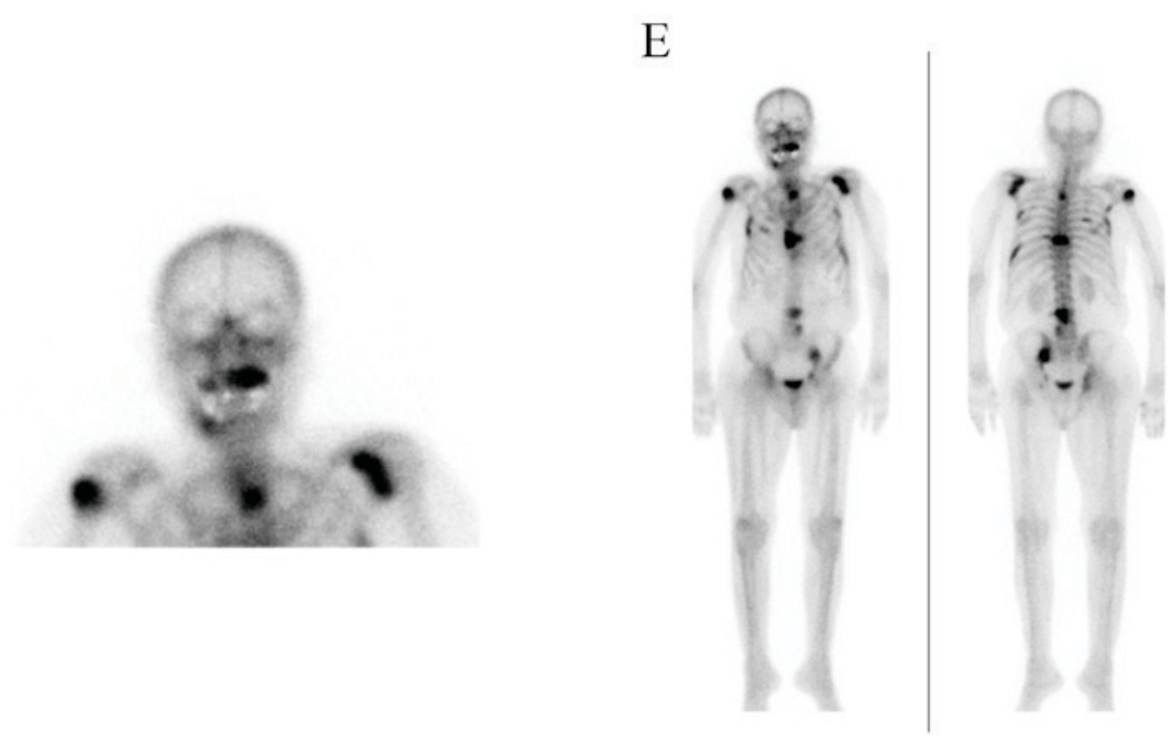

Figure 2. Imaging findings. (A) A panoramic radiograph revealed radiolucencies with diffuse irregularity in the left maxilla and around the apices of the left upper premolar teeth (indicated by the arrowheads). (B) An axial CT (bone window setting) identified an area of low density in the left posterior maxilla and the destruction of the buccal cortical bone (indicated by the arrowhead). (C) A coronal CT (bone window setting) demonstrated bone sequestrum in the left maxilla (indicated by the arrowhead), a perforation in the inferior wall of the maxillary sinus and sinusitis. Bone scintigraphy revealed (D) increased uptake of ${ }^{99 \mathrm{~m}} \mathrm{Tc}-\mathrm{MDP}$ from the left maxilla to part of the sinus, providing evidence of active bone turnover, and (E) uptake of ${ }^{99 \mathrm{~m}} \mathrm{Tc}-\mathrm{mMDP}$ at the upper right humerus, left scapula, sternum, chest and lumbar spine, ribs, pelvic bone, indicating multiple metastatic lesions in the bone. CT, computed tomography.

A

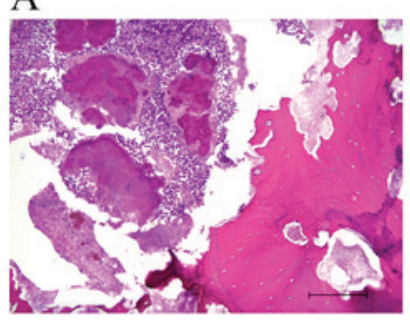

B

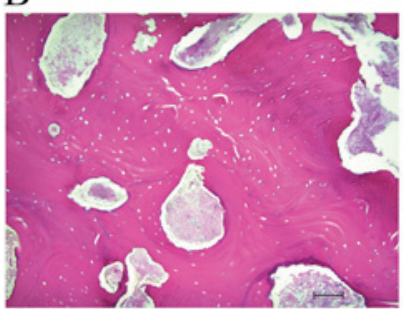

Figure 3. Histological findings from hematoxylin and eosin staining. (A) Histological examination revealed necrotic bone with adherent bacteria and actinomycotic colonies. Scale bar, $200 \mu \mathrm{m}$. (B) No osteoblasts, osteocytes or osteoblasts were observed. Scale bar, $100 \mu \mathrm{m}$.

is called medication-related ONJ (MRONJ) (2). Although the etiology and pathophysiology of MRONJ remain unclear, several potential mechanisms affecting the risk of developing ONJ have been proposed. Hypotheses include the inhibition of osteoclastic bone resorption and remodeling, inflammation and infection, inhibition of angiogenesis, soft tissue toxicity and immune dysfunction (2).

To elucidate the relationship between denosumab use and the development of ONJ, the results of clinical trials presenting

denosumab-related ONJ were reviewed. Typically, the patients with metastatic bone cancer received monthly $120 \mathrm{mg}$ denosumab injections (for 6-36 months) and ONJ was observed at a rate of $0-5 \%$ (Table I) (3-10). By contrast, the patients with osteoporosis typically received $60 \mathrm{mg}$ denosumab injections every 6 months (for 12-36 months) and ONJ was observed at the rate of $0 \%(25-27)$. In a previous report, the prevalence of BRONJ was $\leq 10 \%$ following intravenous BP treatment for malignancy-associated bone disease and $<0.05 \%$ following oral BP treatment for osteoporosis (28). The greater prevalence of BRONJ in patients with malignancy was thought to be associated with the greater and more frequent dosing of BP (29). Thus, the high risk of ONJ in patients with cancer receiving denosumab may also be attributable to the dose and frequency of administration.

The risk of ONJ following treatment with denosumab or BP (including zoledronate, parmidronate or ibandronate) in clinical trials was reviewed (Table II) $(3,4,6-8,10)$. In patients with cancer, the prevalence of denosumab-related ONJ was 0-2\% compared with $0-1.4 \%$ for BRONJ. These reports demonstrated that the prevalence of ONJ in patients treated with denosumab compared with BP is not statistically significant. Although denosumab and $\mathrm{BP}$ have different mechanisms of action (30), the prevalence 


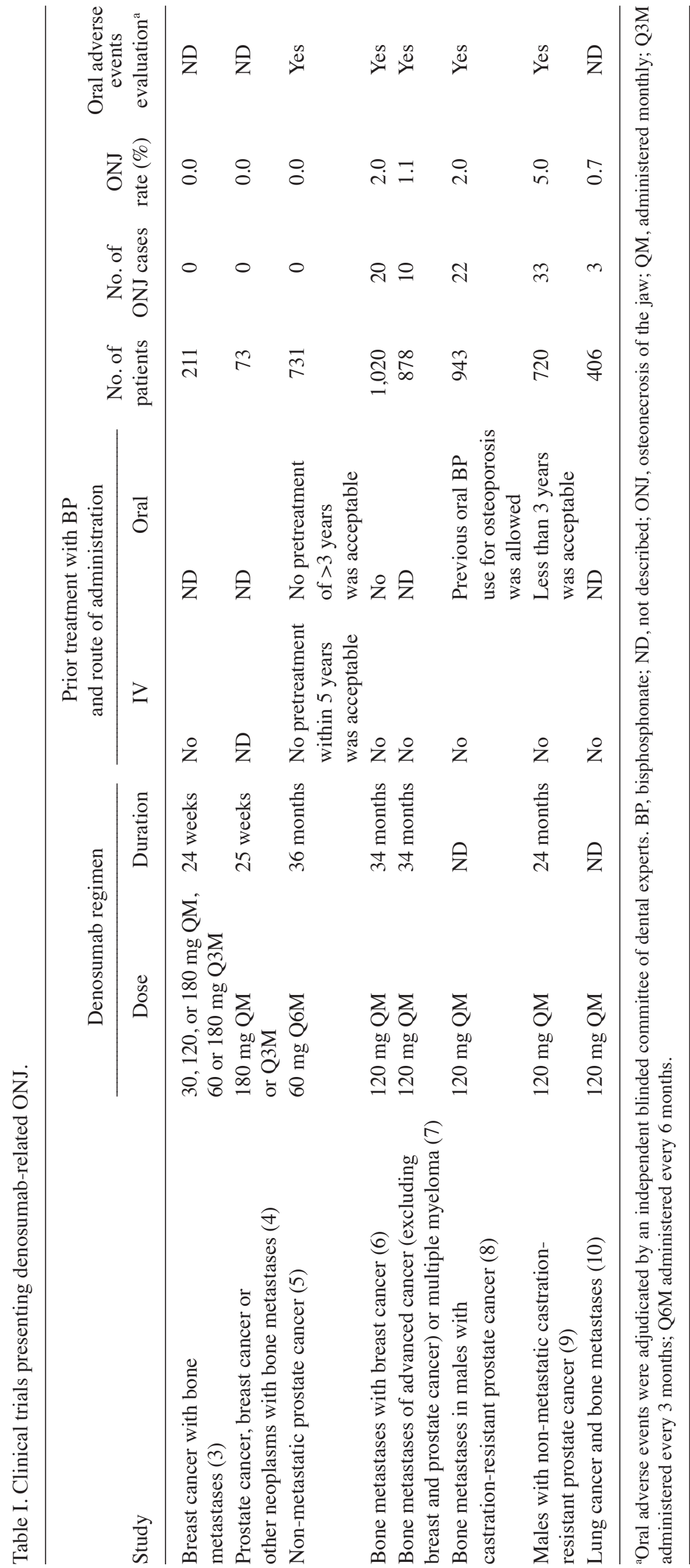




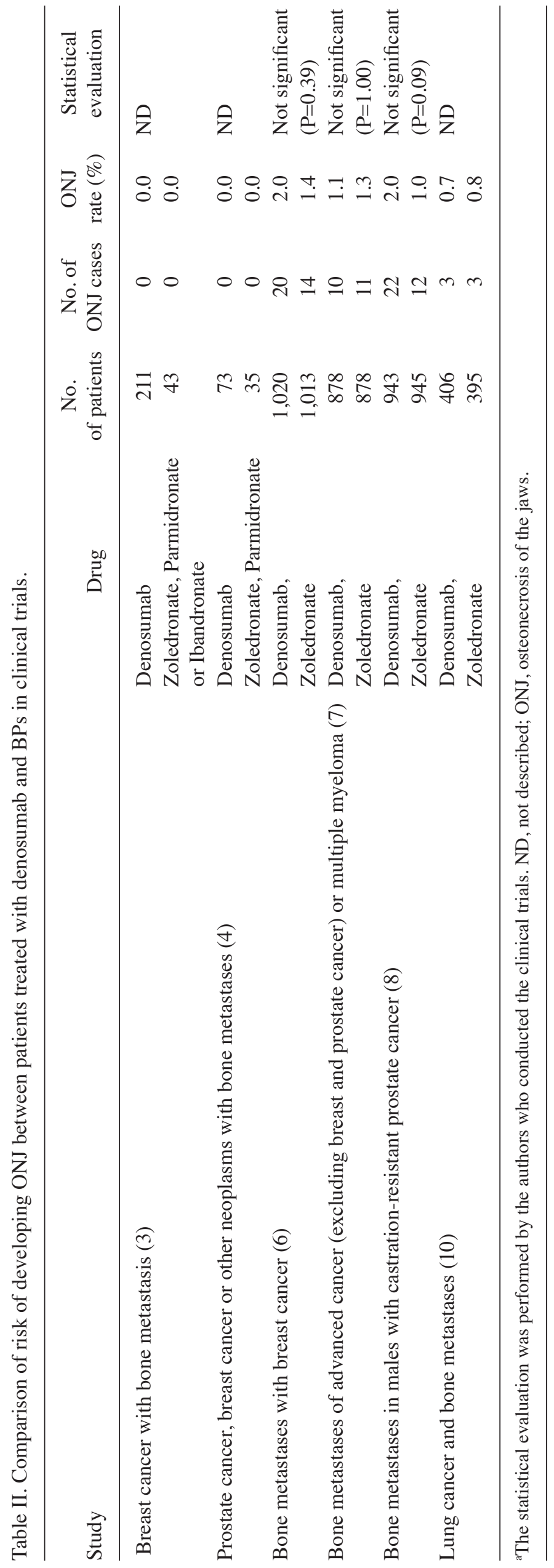


Table III. Reported cases of denosumab-related ONJ in the English language literature $(\mathrm{n}=26)$.

\begin{tabular}{lc}
\hline Characteristics & Value \\
\hline
\end{tabular}

Age, range (mean), years

$49-86(65)$

Gender ratio, M:F

$1.4: 1$

Type of disease, no. of patients

Prostatic cancer

Breast cancer

Colorectal cancer

Lung cancer

9

1

1

Denosumab regimen

Dose, $\mathrm{mg}$, no. of patients $\left(\mathrm{n}=25^{\mathrm{a}}\right)$

120

60

Administration time, range (mean)

months $\left(\mathrm{n}=22^{\mathrm{a}}\right)$

2-36 (13)

Risk factors for ONJ, no. of patients

Systemic factors $\left(n=23^{a}\right)$

Diabetes

Obesity

Smoking

Medications $\left(n=23^{a}\right)$

Chemotherapy

Corticosteroid therapy

Prior BP treatment $\left(n=9^{a}\right)$

Local factors $\left(n=25^{\mathrm{a}}\right)$

Tooth extraction

Apical periodontitis

Clinical manifestations, no. of patients $\left(n=26^{a}\right)$

Mandible

Maxilla

Maxilla and mandible

Symptom, no. of patients $\left(n=26^{a}\right)$

Bone exposure

Pain

Redness

Pus discharge

Swelling

Tenderness

Fistula

Duration of symptoms, range (mean)

months $\left(\mathrm{n}=19^{\mathrm{a}}\right)$

Treatment, no. of patients

Conservative treatment $\left(n=23^{a}\right)$

Antibiotics

Mouth rinse

Surgical treatment $\left(n=16^{a}\right)$

Removal of necrotic bone

Debridement

Incision and drainage

Tooth extraction
Table III. Continued.

\begin{tabular}{lc}
\hline Characteristics & Value \\
\hline $\begin{array}{l}\text { Cessation of denosumab }\left(\mathrm{n}=22^{\mathrm{a}}\right) \\
\text { Yes }\end{array}$ & 19 \\
No & 3 \\
Prognosis, no. of patients $\left(\mathrm{n}=24^{\mathrm{a}}\right)$ & 12 \\
$\quad$ Healed & 12 \\
$\quad$ Unhealed & \\
\hline anocumented cases in the literature $(11-21)$. M, male; F, female; BP, \\
bisphosphonate; ND, not described; ONJ, osteonecrosis of the jaw. \\
\hline
\end{tabular}

of ONJ was similar in patients treated with either. Qi et al (31) reported the risk of denosumab-related ONJ in patients with cancer in a meta-analysis of seven randomized control trials. The overall prevalence of ONJ in patients with cancer receiving denosumab was $1.7 \%$ (31). This analysis demonstrated that the use of denosumab was associated with an increased risk of developing $\mathrm{ONJ}$ when compared with BP treatment or a placebo; however, the increased risk was not statistically significant between denosumab and BP treatment (31).

In the review of the clinical trials conducted in the present study, certain problems in the evaluation of the prevalence of denosumab-related ONJ were concerning. Firstly, several studies included patients that were currently receiving or had previously received oral $\mathrm{BP}(5,8,9)$. BP is an etiopathological factor for BRONJ and the effect of BP on denosumab-related ONJ was not sufficiently discussed. Secondly, in the majority of the trials reviewed, oral adverse events were adjudicated by an independent blinded committee of dental experts, but it was unclear how the oral evaluations were performed prior to and after denosumab treatment $(3,4,10)$. Thirdly, it has been estimated that $\sim 30 \%$ of patients with ONJ do not demonstrate bone exposure (32) and are defined as having stage 0 ONJ (2). These patients may be undiagnosed and not included in the prevalence statistics. Therefore, the actual prevalence of denosumab-related ONJ may be higher compared with that reported in the clinical trials.

To gain a better understanding of the clinical characteristics and management of denosumab-related ONJ, case reports were reviewed (Table III) (11-21). Since 2010, 26 well-documented cases of denosumab-related ONJ have been reported in the English language literature. The reported data includes characteristics of the patients (age, gender, disease), denosumab regimen (dose, administration times), risk factors (systemic factors, medications, experience of BP treatment, local factors) $(1,2,22,23,33,34)$, clinical manifestations (site, symptoms, duration), treatments (conservative and surgical treatment, cessation of denosumab) and prognosis.

In regards to the characteristics of the patients in these case reports, the mean age of the patients with denosumab-related ONJ was 65 years (range, 49-86 years). Among the patients, $24(92 \%)$ were aged $>55$ years and only 2 patients were aged $<55$ years. Denosumab-related ONJ was observed with a predilection for males (male: female ratio, 1.4:1.0). Denosumab-related ONJ was reported in 15 patients (58\%) with 
prostatic cancer, 9 patients (34\%) with breast cancer, 1 patient with colorectal cancer (4\%) and 1 patient with lung cancer (4\%). The regimen of denosumab treatment was reported in 25 of these patients and was typically $120 \mathrm{mg}$ monthly injections.

The risk factors for ONJ in these case reports were examined in the present study, including systemic factors, medications, prior BP treatment and local factors. In the systemic factors, diabetes $(n=2)$, obesity $(n=1)$ and smoking $(n=1)$ were reported. In regards to medication, 8 patients were treated with chemotherapy and 6 were treated with corticosteroid therapy. A prior history of BP treatment was specified for 2 patients, and the 2 patients additionally had past zoledronate treatment. For local factors, a history of dental procedures or conditions was reported in 25 patients. Of these patients, ONJ developed following tooth extraction in 14 patients $(54 \%)$ and apical periodontitis in 2 patients $(8 \%)$. ONJ most frequently occurred at the site of the dental procedure, but 6 cases occurred spontaneously without a dental procedure. In the review of clinical manifestations, the mandible was the most commonly affected site $(n=17)$, followed by the maxilla $(n=6)$, and 3 patients developed ONJ of the maxilla and mandible. Symptoms of ONJ were reported in 26 patients, including bone exposure $(n=25)$, pain $(n=9)$, redness $(n=5)$, pus-containing discharge $(n=4)$, swelling $(n=4)$, tenderness $(n=2)$ and fistula $(n=2)$. The duration of symptoms was reported in 19 cases, with a mean duration of 5 months (range, 0.25-12 months).

In regards to the treatments for ONJ that these patients received, conservative treatments were reported in 23 patients [administration of antibiotics in 22 patients (96\%) and a mouth rinse in 21 patients $(91 \%$ ]. A total of $14(54 \%)$ were also treated surgically. The surgical treatments for ONJ included the removal of necrotic bone $(n=12)$, debridement $(n=5)$, incision and drainage $(n=1)$ and tooth extraction $(n=1)$. Descriptions about the cessation of denosumab were found for 22 patients; 19 patients $(86 \%)$ had their denosumab administration discontinued and 3 patients (14\%) preferred to continue denosumab therapy. The prognosis was described in 24 patients; 12 patients healed, but the other 12 patients did not.

In the present review of the reported cases, denosumab-related ONJ had a similar clinical presentation to BRONJ $(22,23)$. There was a tendency for ONJ to develop in the mandible in elderly patients. In addition, dental extraction or inflammatory dental disease was an important initiating factor. A complex medical history was also suspected to affect the prevalence of ONJ.

Denosumab-related ONJ was primarily observed in patients with prostate cancer. Qi et al (31) reported an analysis of ONJ based on tumor type, demonstrating that the prevalence of denosumab-related ONJ in patients with prostate cancer was higher compared with that in patients with non-prostate cancers. In their analysis, the median follow-up period for prostate cancer was longer compared with that for non-prostate cancer (31). The authors suggested that the variability in the prevalence of ONJ in the different cancer types may be due to this variation, as it is probable that the prevalence of ONJ would have increased with a longer follow-up period (31). A similar phenomenon has been observed in clinical trials, which demonstrated a trend toward cumulative increases in ONJ over time in patients with cancer (6,7). In addition, the relatively long clinical course of prostate cancer (35) may affect the prevalence of denosumab-related ONJ. In the review conducted in the present study, ONJ was also observed prevalently in males. This is likely due to the fact that denosumab-related ONJ was most frequently observed in patients with prostate cancer.

Denosumab has produced excellent clinical results compared with BP for the treatment of patients with bone-associated cancer and osteoporosis, causing great increases in bone mineral density and the suppression of bone turnover markers $(3,26)$, in addition to efficacy even in patients who were previously resistant to BP treatment (36). In the review of case reports conducted in the present study, 2 patients transitioned from BP and developed denosumab-related ONJ. Thus, the effect of prior BP treatment should be considered as an etiopathological factor for denosumab-related ONJ. In the case reported in the present study, the patient received a single zoledronate treatment followed by denosumab therapy. Although the short duration of BP treatment in this patient was unlikely to have contributed to the development of ONJ, the possibility that BP treatment may synergistically enhance the denosumab-related inhibition of osteoclastic activity and development of ONJ should be considered.

In the literature review performed in the present study, no clear relationship between the duration of denosumab treatment and the development of denosumab-related ONJ was observed. In one case, a patient developed ONJ after receiving two $60 \mathrm{mg}$ doses of denosumab (14), while in another, a patient developed ONJ after 36 monthly administrations of $120 \mathrm{mg}$ denosumab (21). Due to the limited number of patients included in the present review, the accurate assessment of the association between the dose/duration of denosumab treatment and the onset of ONJ was difficult to determine. This association should be examined in a larger patient cohort.

The concept of a 'drug holiday' in patients receiving denosumab who require invasive dental treatments is an ongoing area of controversy (2). There are no previous studies to support or refute the strategy of stopping denosumab therapy for the prevention or treatment of MRONJ (2). In the review conducted in the present study, 3 patients continued on denosumab and administration was stopped in 19 patients following MRONJ. Otto et al (37) reported 2 successful cases of surgical treatment of denosumab-related ONJ. The authors recommended that any surgical intervention should be withheld until $\geq 4$ months after cessation of denosumab treatment (37). By contrast, Diz et al (12) reported a case of denosumab-related ONJ due to tooth extraction 6 months after cessation of denosumab therapy. Notably, Vyas et al (38) reported a case of denosumab-related ONJ that healed within 1 month of cessation of denosumab. Thus, it is difficult to determine the appropriate period of a drug holiday for denosumab.

$\mathrm{BP}$ is incorporated into the mineral matrix of the bone, but denosumab has no binding affinity for the bone matrix (14). In terms of drug half-life, denosumab has an advantage compared with BP, with a shorter half-life of 25.4 days compared with 10-12 years, respectively (39). Thus, ONJ may resolve more rapidly after a drug holiday in patients taking denosumab compared with patients taking BP. Based on the pharmacokinetics of denosumab, it may be possible to place patients on an effective drug holiday prior to surgical interventions to promote bone healing. In the evaluation of bone metabolism, 
the measurement of bone turnover markers aids in the assessment of the patient's condition (40). Further studies using bone turnover markers are required to verify the rate of bone turnover after cessation of denosumab in patients who develop, or are at significant risk of developing, denosumab-related ONJ. Discontinuing bone antiresorptive agent therapy is an important part of the management of bone conditions (41). The injudicious discontinuation of denosumab therapy can lead to an increased risk of the progression of bony lesions in patients with cancer. Thus, sufficient medical consultation and discussion are appropriate prior to the cessation of denosumab treatment.

In patients with cancer that develop ONJ, the current recommendations are conservative treatment and to avoid additional invasive surgeries (2). In a previous report, conservative treatment (oral monitoring, oral rinses, antibiotics) led to an overall ONJ resolution rate of $36 \%$ (42). The resolution rate was greater in the denosumab-treated group (40.4\%) compared with the zoledronate-treated group (29.7\%) (42). This more rapid recovery is likely due to the more rapidly reversible effects of denosumab on bone turnover compared with BP (42).

Conservative therapy may not always lead to the complete resolution of lesions, but it can provide long-term symptomatic relief (43). In the case reported in the present study, the patient refused radical surgical treatment under general anesthesia and was treated with a minimal debridement of the necrotic bone followed by chlorhexidine mouth rinses and antibiotic therapy. Although the lesion did not heal, the symptoms disappeared or were manageable. The patient was satisfied with the results of the treatment, and their condition was maintained throughout the long follow-up period.

Although treatment guidelines supports the conservative management of ONJ $(2,23)$, in the review of the literature conducted in the present study, certain lesions did not respond well to conservative treatment and were thus managed surgically. Ristow et al (44) reported treatment outcomes for MRONJ, with the success rates of conservative treatment regimens ranging from between $<20 \%$ and $>50 \%$, which is significantly lower compared with the success rates of $>85 \%$ reported for surgical approaches (44). The authors suggested that surgical interventions should be considered for all stages of MRONJ (44). Although surgery is an option for the resolution of non-responsive ONJ lesions, certain cases that were reviewed in the present study did not heal despite surgical treatments $(14,15)$. These results indicate that the appropriate treatment plan for ONJ must be decided in accordance with the situation and condition of the individual patient.

In the present case report, Actinomyces colonies were observed in the histological examination, but not in the microbiological analysis. Recent studies have revealed the presence of Actinomyces colonies in biopsy material from patients with MRONJ, suggesting that these bacteria contribute to MRONJ-associated infections $(2,45,46)$. Members of the Actinomyces genus are filamentous, gram-positive and anaerobic bacteria that typically colonize the mouth, colon and vagina. The most common disease caused by these bacteria is cervicofacial actinomycosis (47), which presents as a soft tissue induration with abscesses and multiple fistula. Histopathologically, Actinomyces is identified primarily by its characteristic formation of sulfur granules (45). The diagnosis of actinomycosis is based on microbiological and/or histological analysis $(48,49)$. The results of the two examinations often differ from one another (49). The microbiological identification of Actinomyces is difficult because the anaerobic processing of the specimens is strict (48), requiring careful culture, preferably when the patient has received no antibiotics for 7-10 days (50). Furthermore, the growth rate of Actinomyces bacteria is slow (48). Thus, false negative results are frequently caused by the taking or transporting of specimens for studies (49). By contrast, the tissue samples for histological examination do not require any special treatment and the diagnosis of actinomycosis relies only on the detection of sulfur granules (48). Therefore, the final diagnosis has a tendency to depend on histopathological confirmation (51). This explains the discrepancy in the results of the case reported in the current study. It remains unclear whether Actinomyces organisms trigger MRONJ or whether MRONJ triggers secondary infection with these bacteria. The role of these organisms in the development MRONJ requires further investigation.

In conclusion, the prevalence of denosumab-related ONJ is low, but remains potentially severe complication of denosumab treatment. Thus, the management of denosumab-related ONJ represents a challenge for clinicians. Since denosumab may offer superior results compared with BP for the treatment of metastatic cancer to the bone or osteoporosis, the use of denosumab is expected to increase in the near future. Clinicians should also be aware of the recognized risk factors for denosumab-related ONJ, in order to aid in its diagnosis. The results of the current study indicate that patients treated with denosumab should receive prophylactic treatment to maintain their oral health prior to, during and after denosumab treatment. Invasive dental procedures should also be avoided during and after denosumab use. If a patient develops denosumab-related ONJ, conservative therapy is the mainstay; however, surgery is an option for non-responsive ONJ lesions. The contribution of denosumab to the development of ONJ remains unclear; further studies specifically designed to assess the prevalence of ONJ in larger cohorts over long observation periods are required. Further research on animal models is also needed to elucidate the underlying mechanism of denosumab-related ONJ. Analyses of the required length of drug holiday and the timing of surgical intervention in denosumab-related ONJ by measuring the levels of bone turnover markers are warranted. Further studies remain necessary to establish guidelines for the prevention and effective treatment of denosumab-related ONJ.

\section{References}

1. Yamashita J and McCauley LK: Antiresorptives and osteonecrosis of the jaw. J Evid Based Dent Pract 12 (3 Suppl): S233-S247, 2012.

2. Ruggiero SL, Dodson TB, Fantasia J, Goodday R, Aghaloo T, Mehrotra B and O'Ryan F; American Association of Oral and Maxillofacial Surgeons: American Association of Oral and Maxillofacial Surgeons position paper on medication-related osteonecrosis of the jaw-2014 update. J Oral Maxillofac Surg 72: 1938-1956, 2014.

3. Lipton A, Steger GG, Figueroa J, Alvarado C, Solal-Celigny P, Body JJ, de Boer R, Berardi R, Gascon P, Tonkin KS, et al: Randomized active-controlled phase II study of denosumab efficacy and safety in patients with breast cancer-related bone metastases. J Clin Oncol 25: 4431-4437, 2007. 
4. Fizazi K, Lipton A, Mariette X, Body JJ, Rahim Y, Gralow JR, Gao G, Wu L, Sohn W and Jun S: Randomized phase II trial of denosumab in patients with bone metastases from prostate cancer, breast cancer, or other neoplasms after intravenous bisphosphonates. J Clin Oncol 27: 1564-1571, 2009.

5. Smith MR, Egerdie B, Hernández Toriz N, Feldman R, Tammela TL, Saad F, Heracek J, Szwedowski M, Ke C, Kupic A, et al: Denosumab in men receiving androgen-deprivation therapy for prostate cancer. N Engl J Med 361: 745-755, 2009.

6. Stopeck AT, Lipton A, Body JJ, Steger GG, Tonkin K, de Boer RH, Lichinitser M, Fujiwara Y, Yardley DA, Viniegra M, et al: Denosumab compared with zoledronic acid for the treatment of bone metastases in patients with advanced breast cancer: A randomized, double-blind study. J Clin Oncol 28: 5132-5139, 2010.

7. Henry DH, Costa L, Goldwasser F, Hirsh V, Hungria V, Prausova J, Scagliotti GV, Sleeboom H, Spencer A, Vadhan-Raj S, et al: Randomized, double-blind study of denosumab versus zoledronic acid in the treatment of bone metastases in patients with advanced cancer (excluding breast and prostate cancer) or multiple myeloma. J Clin Oncol 29: $1125-1132,2011$

8. Fizazi K, Carducci M, Smith M, Damião R, Brown J, Karsh L, Milecki P, Shore N, Rader M, Wang H, et al: Denosumab versus zoledronic acid for treatment of bone metastases in men with castration-resistant prostate cancer: A randomised, double-blind study. Lancet 377: 813-822, 2011.

9. Smith MR, Saad F, Coleman R, Shore N, Fizazi K, Tombal B Miller K, Sieber P, Karsh L, Damião R, et al: Denosumab and bone-metastasis-free survival in men with castration-resistant prostate cancer: Results of a phase 3, randomised, placebo-controlled trial. Lancet 379: 39-46, 2012

10. Scagliotti GV, Hirsh V, Siena S, Henry DH, Woll PJ, Manegold C, Solal-Celigny P, Rodriguez G, Krzakowski M, Mehta ND, et al: Overall survival improvement in patients with lung cancer and bone metastases treated with denosumab versus zoledronic acid: Subgroup analysis from a randomized phase 3 study. J Thorac Oncol 7: 1823-1829, 2012

11. Taylor KH, Middlefell LS and Mizen KD: Osteonecrosis of the jaws induced by anti-RANK ligand therapy. Br J Oral Maxillofac Surg 48: 221-223, 2010.

12. Diz P, López-Cedrún JL, Arenaz J and Scully C: Denosumab-related osteonecrosis of the jaw. J Am Dent Assoc 143: 981-984, 2012

13. Malan J, Ettinger K, Naumann E and Beirne OR: The relationship of denosumab pharmacology and osteonecrosis of the jaws Oral Surg Oral Med Oral Pathol Oral Radiol 114: 671-676, 2012.

14. Pichardo SE, Kuypers SC and van Merkesteyn JP: Denosumab osteonecrosis of the mandible: A new entity? A case report. J Craniomaxillofac Surg 41: e65-e69, 2013.

15. Olate S, Uribe F, Martinez F, Almeida A and Unibazo A Osteonecrosis of the jaw in patient with denosumab therapy. Int J Clin Exp Med 7: 3707-3709, 2014.

16. O'Halloran M, Boyd NM and Smith A: Denosumab and osteonecrosis of the jaws-the pharmacology, pathogenesis and a report of two cases. Aust Dent J 59: 516-519, 2014.

17. Ohga N, Yamazaki Y, Tsuboi K and Kitagawa Y: Healing of osteonecrosis of the jaw (ONJ) after discontinuation of denosumab in a patient with bone metastases of colorectal cancer: A case report and hypothesis. Quintessence Int 46: 621-626, 2015.

18. You Tm, Lee KH, Lee SH and Park W: Denosumab-related osteonecrosis of the jaw: A case report and management based on pharmacokinetics. Oral Surg Oral Med Oral Pathol Oral Radiol 120: 548-553, 2015

19. Matsushita Y, Hayashida S, Morishita K, Sakamoto H, Naruse T, Sakamoto Y, Yamada SI, Yanamoto S, Fujita S, Ikeda T and Umeda M: Denosumab-associated osteonecrosis of the jaw affects osteoclast formation and differentiation: Pathological features of two cases. Mol Clin Oncol 4: 191-194, 2016.

20. Yamagata K, Nagai H, Baba O, Uchida F, Kanno N, Hasegawa S Yanagawa $\mathrm{T}$ and Bukawa $\mathrm{H}$ : A case of brain abscess caused by medication-related osteonecrosis of the jaw. Case Rep Dent 2016: 7038618, 2016.

21. Owosho AA, Blanchard A, Levi L, Kadempour A, Rosenberg H, Yom SK, Farooki A, Fornier M, Huryn JM and Estilo CL: Osteonecrosis of the jaw in patients treated with denosumab for metastatic tumors to the bone: A series of thirteen patients. J Craniomaxillofac Surg 44: 265-270, 2016.
22. Advisory Task Force on Bisphosphonate-Related Ostenonecrosis of the Jaws, American Association of Oral and Maxillofacial Surgeons: American Association of Oral and Maxillofacial Surgeons position paper on bisphosphonate-related osteonecrosis of the jaws. J Oral Maxillofac Surg 65: 369-376, 2007.

23. Ruggiero SL, Dodson TB, Assael LA, Landesberg R, Marx RE and Mehrotra B; American Association of Oral and Maxillofacial Surgeons: American Association of Oral and Maxillofacial Surgeons position paper on bisphosphonate-related osteonecrosis of the jaws-2009 update. J Oral Maxillofac Surg 67 (5 Suppl): S2-S12, 2009.

24. Marx RE: Pamidronate (Aredia) and zoledronate (Zometa) induced avascular necrosis of the jaws: A growing epidemic. J Oral Maxillofac Surg 61: 1115-1117, 2003

25. Cummings SR, San Martin J, McClung MR, Siris ES, Eastell R, Reid IR, Delmas P, Zoog HB, Austin M, Wang A, et al: Denosumab for prevention of fractures in postmenopausal women with osteoporosis. N Engl J Med 361: 756-765, 2009.

26. Roux C, Hofbauer LC, Ho PR, Wark JD, Zillikens MC, Fahrleitner-Pammer A, Hawkins F, Micaelo M, Minisola S, Papaioannou N, et al: Denosumab compared with risedronate in postmenopausal women suboptimally adherent to alendronate therapy: Efficacy and safety results from a randomized open-label study. Bone 58: 48-54, 2014.

27. Nakamura T, Matsumoto T, Sugimoto T, Hosoi T, Miki T, Gorai I, Yoshikawa H, Tanaka Y, Tanaka S, Sone T, et al: Clinical trials express: Fracture risk reduction with denosumab in Japanese postmenopausal women and men with osteoporosis: Denosumab fracture intervention randomized placebo controlled trial (DIRECT). J Clin Endocrinol Metab 99: 2599-2607, 2014.

28. Dinca O, Bucur MB, Bodnar D, Vladan C and Bucur A: Extensive osteonecrosis of the mandible after therapy with denosumab following bisphosphoantes therapy. Acta Endo 10: 457-462, 2014

29. Capozzi A, Lello S and Pontecorvi A: The inhibition of RANK-ligand in the management of postmenopausal osteoporosis and related fractures: The role of denosumab. Gynecol Endocrinol 30: 403-408, 2014.

30. Baron R, Ferrari S and Russell RG: Denosumab and bisphosphonates: Different mechanisms of action and effects. Bone 48: 677-692, 2011.

31. Qi WX, Tang LN, He AN, Yao Y and Shen Z: Risk of osteonecrosis of the jaw in cancer patients receiving denosumab: A meta-analysis of seven randomized controlled trials. Int J Clin Oncol 19: 403-410, 2014.

32. Fedele S, Porter SR, D'Aiuto F, Aljohani S, Vescovi P,Manfredi M, Arduino PG, Broccoletti R, Musciotto A, Di Fede O, et al: Nonexposed variant of bisphosphonate-associated osteonecrosis of the jaw: A case series. Am J Med 123: 1060-1064, 2010.

33. Campisi G, Fedele S, Fusco V, Pizzo G, Di Fede O and Bedogni A: Epidemiology, clinical manifestations, risk reduction and treatment strategies of jaw osteonecrosis in cancer patients exposed to antiresorptive agents. Future Oncol 10: 257-275, 2014.

34. Patel V, McLeod NM, Rogers SN and Brennan PA: Bisphosphonate osteonecrosis of the jaw-a literature review of UK policies versus international policies on bisphosphonates, risk factors and prevention. Br J Oral Maxillofac Surg 49: 251-257, 2011.

35. Coleman RE: Metastatic bone disease: Clinical features, pathophysiology and treatment strategies. Cancer Treat Rev 27: 165-176, 2001.

36. Body JJ, Lipton A, Gralow J, Steger GG, Gao G, Yeh H and Fizazi K: Effects of denosumab in patients with bone metastases with and without previous bisphosphonate exposure. J Bone Miner Res 25: 440-446, 2010.

37. Otto S, Baumann S, Ehrenfeld M and Pautke C: Successful surgical management of osteonecrosis of the jaw due to RANK-ligand inhibitor treatment using fluorescence guided bone resection. J Craniomaxillofac Surg 41: 694-698, 2013.

38. Vyas S, Hameed S and Murugaraj V: Denosumab-associated osteonecrosis of the jaw-a case report. Dent Update 41: 449-450, 2014.

39. Uyanne J, Calhoun CC and Le AD: Antiresorptive drug-related osteonecrosis of the jaw. Dent Clin North Am 58: 369-384, 2014

40. Scott LJ and Muir VJ: Denosumab: In the prevention of skeletal-related events in patients with bone metastases from solid tumours. Drugs 71: 1059-1069, 2011.

41. Epstein MS, Ephros HD and Epstein JB: Review of current literature and implications of RANKL inhibitors for oral health care providers. Oral Surg Oral Med Oral Pathol Oral Radiol 116: e437-e442, 2013. 
42. Saad F, Brown JE, Van Poznak C, Ibrahim T, Stemmer SM Stopeck AT, Diel IJ, Takahashi S, Shore N, Henry DH, et al: Incidence, risk factors, and outcomes of osteonecrosis of the jaw: Integrated analysis from three blinded active-controlled phase III trials in cancer patients with bone metastases. Ann Oncol 23: 1341-1347, 2012.

43. Khan AA, Morrison A, Hanley DA, Felsenberg D, McCauley LK, O'Ryan F, Reid IR, Ruggiero SL, Taguchi A, Tetradis S, et al: Diagnosis and management of osteonecrosis of the jaw: A systematic review and international consensus. J Bone Miner Res 30: 3-23, 2015.

44. Ristow O, Otto S, Troeltzsch M, Hohlweg-Majert B and Pautke C: Treatment perspectives for medication-related osteonecrosis of the jaw (MRONJ). J Craniomaxillofac Surg 43: 290-293, 2015.

45. Schipmann S, Metzler P, Rössle M, Zemann W, von Jackowski J, Obwegeser JA, Grätz KW and Jacobsen C: Osteopathology associated with bone resorption inhibitors-which role does Actinomyces play? A presentation of 51 cases with systematic review of the literature. J Oral Pathol Med 42: 587-593, 2013.

46. Katsarelis H, Shah NP, Dhariwal DK and Pazianas M: Infection and medication-related osteonecrosis of the jaw. J Dent Res 94: 534-539, 2015.
47. Miller M and Haddad AJ: Cervicofacial actinomycosis. Oral Surg Oral Med Oral Pathol Oral Radiol Endod 85: 496-508, 1998.

48. Hansen T, Kunkel M, Kirkpatrick CJ and Weber A: Actinomyces in infected osteoradionecrosis-underestimated? Hum Pathol 37: 61-67, 2006.

49. Bartkowski SB, Zapala J, Heczko P and Szuta M: Actinomycotic osteomyelitis of the mandible: Review of 15 cases. J Craniomaxillofac Surg 26: 63-67, 1998.

50. Goldberg MH: Diagnosis and treatment of cervicofacial actinomycosis. Oral Maxillofac Surg Clin North Am 15: 51-58, 2003.

51. Kuyama K, Sun Y, Fukui K, Maruyama S, Ochiai E, Fukumoto M, Ikeda N, Kondoh T, Iwadate K, Takagi R, et al: Tumor mimicking actinomycosis of the upper lip: Report of two cases. Oral Med Pathol 15: 95-99, 2011. 